\title{
HOW WERE THE HILBERT-EINSTEIN EQUATIONS DISCOVERED?
}

\author{
A.A. Logunov, M.A.Mestvirishvili, V.A. Petrov ${ }^{1}$ \\ Division of Theoretical Physics, \\ Institute for High Energy Physics, \\ 142281, Protvino, Moscow Region, Russian Federation
}

\begin{abstract}
The pathways along which A. Einstein and D. Hilbert independently came to the gravitational field equations are traced. Some of the papers that assert a point of view on the history of the derivation of the gravitational field equations "that radically differs from the standard point of view" are critically analyzed. It is shown that the conclusions drawn in these papers are completely groundless.
\end{abstract}

\section{Introduction}

Since the studies by J. Earman and C. Glymour [1] it became clear that the equations of A. Einstein's general relativity were discovered almost simultaneously, but with different methods, by D. Hilbert and A. Einstein.

In 1997 an article appeared in the journal "Science" under the title "Belated Decision in the Hilbert-Einstein Priority Dispute" [2], the authors of which claim that "...knowledge of Einstein's result may have been crucial to Hilbert's introduction of the trace term into his field equations". On this ground they push forward their point of view "that radically differs from the standard point of view" and which is exposed in a many-page ref.[3].

According to the standard point of view Einstein and Hilbert, independently of each other and in different ways, discovered the gravitational field equations. The same question was the subject of the paper [4].

What is the question? In the Einstein paper [5] the gravitational field equations are given:

$$
\sqrt{-g} R_{\mu \nu}=-\varkappa\left(T_{\mu \nu}-\frac{1}{2} g_{\mu \nu} T\right),
$$

\footnotetext{
${ }^{1}$ e-mail: Vladimir.Petrov@ihep.ru
} 
where, as usual, $g_{\mu \nu}$ is a metric tensor; $R_{\mu \nu}$ is the Ricci tensor, $\varkappa$ stands for the gravitational coupling constant, $T_{\mu \nu}$ is the energy-momentum tensor density for matter, $T$ is the trace of $T_{\mu \nu}$ :

$$
T=g^{\mu \nu} T_{\mu \nu}
$$

The authors of the paper [2] assert that Hilbert, when having taken knowledge of these equations and having seen the "trace term" $\left(\frac{1}{2} g_{\mu \nu} T\right)$, would be also "introduced" into his equations [6],

$$
\sqrt{g}\left(R_{\mu \nu}-\frac{1}{2} g_{\mu \nu} R\right)=-\frac{\partial \sqrt{g} L}{\partial g^{\mu \nu}},
$$

the trace term (in this case $\frac{1}{2} g_{\mu \nu} R$, where the trace $R=g^{\mu \nu} R_{\mu \nu}$ ).

Let us see in what field equation Hilbert needed, according to the authors [2], to "introduce the trace term". The authors of ref. [2] do not take into account that in the Hilbert approach nothing can be "introduced" because everything is exactly defined by the world function (Lagrangean).

$$
H=R+L,
$$

discovered by Hilbert, which plays a key role for derivation of the gravitational equations in the framework of the least action principle.

The authors of [2] produced their discovery when they took knowledge of the proofs of the Hilbert paper (in which, by the way, some parts are missed. See [7], where, in particular, the remained parts of the proofs are reproduced) and saw that the gravitational field equations were presented there in the form of the variational derivative of $[\sqrt{g} R]$ in $g^{\mu \nu}$

$$
\frac{\partial \sqrt{g} R}{\partial g^{\mu \nu}}-\partial_{k} \frac{\partial \sqrt{g} R}{\partial g_{k}^{\mu \nu}}+\partial_{k} \partial_{\ell} \frac{\partial \sqrt{g} R}{\partial g_{k \ell}^{\mu \nu}}=-\frac{\partial \sqrt{g} L}{\partial g^{\mu \nu}},
$$

but not in the form (1). Thereof they draw their conclusion that Hilbert did not derived the gravitation equations in the form (1).

But if even everything were so, then at any rate Hilbert needed nothing to "introduce" in addition because Eq.(2) turns exactly into Eq.(1) after some quite trivial calculations. Things, however, go not in such a way as authors of [2] wrote. In order to show that the statement by 
the authors of [2] has no serious grounds we have to give an account of the basics of D. Hilbert's work (see Section 1).

On the basis of the idea of equivalence of acceleration and gravity Einstein in the joint article [8] with M. Grossmann in 1913 identified the gravitational field and the metric tensor of a pseudo-Riemannian (below, just Riemannian) space. In such a way the tensorial gravitational field was introduced. Einstein, in this article, formulates, on the basis of some simple model, the general energy-momentum conservation law:

$$
\partial_{\nu}\left(\sqrt{-g} \Theta_{\sigma}^{\nu}\right)+\frac{1}{2} \sqrt{-g} \Theta_{\mu \nu} \partial_{\sigma} g^{\mu \nu}=0 .
$$

"The first three of these relations $(\sigma=1,2,3)$ express the momentum conservation law, the latter $(\sigma=4)$ that of energy conservation," Einstein wrote. Here $\Theta_{\mu \nu}$ stands for the energy-momentum tensor of matter. It is necessary to note that such a law of energy-momentum conservation for any material system was introduced by Einstein still as a plausible physical assumption. In the same article M. Grossman showed that Eq.(3) is covariant under arbitrary transformations and can be cast into the form

$$
\nabla_{\nu} \Theta_{\sigma}^{\nu}=0
$$

here $\nabla_{\nu}$ is a covariant derivative with respect to the metric $g_{\mu \nu}$.

Einstein posed a problem to find out the gravitational equation of the form

$$
\Gamma_{\mu \nu}=\varkappa \Theta_{\mu \nu},
$$

where $\Gamma_{\mu \nu}$ is a tensor composed of the metric and its derivatives.

It worth to notice that in the part of this article which was written by Grossman, the possible use of the Ricci tensor, $R_{\mu \nu}$, as $\Gamma_{\mu \nu}$ from Eq.(5), was discussed.

Nonetheless M.Grossman finally rejected such a proposal: "It turns out, however, that this tensor in the special case of infinitely weak static force field does not reduce itself into $\Delta \varphi$ ".

Later Einstein, following his ideas, searched for $\Gamma_{\mu \nu}$ as a tensor under arbitrary linear transformations. He would follow this way till November 1915. At the end of June (beginning of July) 1915 Einstein, invited by Hilbert, spent a week in Göttingen, where he, as he recollected later, "gave there six two-hour lectures". It is evident that afterwards D. Hilbert got interested in the problem. 
The Einstein formulation of the problem and his identification of the gravitation potential with the metric tensor $g_{\mu \nu}$ of a Riemannian space appeared the key ones for Hilbert. That was sufficient for him in order to find out the gravitational field equation proceeding from the principle of the least action (Hilbert's Axiom I) and from his profound knowledge of the theory of invariants. All this is directly seen in the paper by Hilbert [6].

Below we give an account of Hilbert's approach to derivation of the gravitational field equation, and also give a critical analysis of the articles $[2,3,4]$ devoted to the same question.

\section{Hilbert's Approach}

Let us consider attentively Hilbert's approach [6]. He formulates Axiom I:

The laws of physical events are defined by the world function $H$ the arguments of which are

$$
\begin{gathered}
g_{\mu \nu}, g_{\mu \nu \ell}=\frac{\partial g_{\mu \nu}}{\partial x^{\ell}}, \quad g_{\mu \nu \ell k}=\frac{\partial^{2} g_{\mu \nu}}{\partial x^{\ell} \partial x^{k}} \\
q_{s}, q_{s \ell}=\frac{\partial q_{s}}{\partial x^{\ell}}, \quad(\ell, k=1,2,3,4),
\end{gathered}
$$

being the variation of the integral

$$
\begin{gathered}
\int H \sqrt{g} d \omega, \\
\left(g=\left|g_{\mu \nu}\right|, \quad d \omega=d x_{1} d x_{2} d x_{3} d x_{4}\right),
\end{gathered}
$$

disappears for any of 14 potentials $g_{\mu \nu}, q_{s} "$.

He writes further: "As to the world function $H$, additional axioms are needed for its unambigious definition. If only second derivatives of potentials $g^{\mu \nu}$ can enter the gravitation equations, then the function $H$ has to have the form ${ }^{2}$

$$
H=R+L,
$$

\footnotetext{
${ }^{2}$ In ref.[6] Hilbert used the notations $K_{\mu \nu}$ and $K$ for the Ricci tensor and the scalar curvature. We use for them, and also for other quantities, modern notations. We also use in all citations the numeration of formulas according to our text.
} 
where $R$ is an invariant following from the Riemann tensor (scalar curvature of a four-dimensional manifold):

$$
\begin{gathered}
R=g^{\mu \nu} R_{\mu \nu} \\
R_{\mu \nu}=\partial_{\nu} \Gamma_{\mu \alpha}^{\alpha}-\partial_{\alpha} \Gamma_{\mu \nu}^{\alpha}+\Gamma_{\mu \alpha}^{\lambda} \Gamma_{\lambda \nu}^{\alpha}-\Gamma_{\mu \nu}^{\lambda} \Gamma_{\lambda \alpha}^{\alpha},
\end{gathered}
$$

and $L$ is a function of variables $g^{\mu \nu}, g_{\ell}^{\mu \nu}, q_{s}, q_{s k}$ only. Besides that, we assume further on that $L$ does not depend on $g_{\ell}^{\mu \nu}$.

From variation in the 10 gravitational potentials the 10 Lagrange differential equations follow

$$
\frac{\partial \sqrt{g} R}{\partial g^{\mu \nu}}-\partial_{k} \frac{\partial \sqrt{g} R}{\partial g_{k}^{\mu \nu}}+\partial_{k} \partial_{\ell} \frac{\partial \sqrt{g} R}{\partial g_{k \ell}^{\mu \nu}}=-\frac{\partial \sqrt{g} L}{\partial g^{\mu \nu}} " .
$$

It is easy to see from (8) and (9) that both in $R$ and $R_{\mu \nu}$ secondorder derivatives of the metric enter linearly. Second rank tensors with such properties are

$$
R_{\mu \nu} \text { and } g_{\mu \nu} R \text {. }
$$

\section{All other tensors with such properties are obtained as combinations of these tensors.}

This conclusion, to some extent, was known to Einstein, and he, mentioning tensors of the second rank, which could lead to the gravitational equations with derivatives not higher than of the second order, wrote in the letter to H.A. Lorentz 19 January 1916 [9]:

“... aside from tensors...

$$
R_{\mu \nu} \quad \text { and } \quad g_{\mu \nu} R
$$

there are no (arbitrary substitutions for covariant) tensors..."

For mathematician D. Hilbert that was evident.

Let us denote for the sake of brevity and following to Hilbert the left part of the equation by the symbol

$$
[\sqrt{g} R]_{\mu \nu}=\frac{\partial \sqrt{g} R}{\partial g^{\mu \nu}}-\partial_{k} \frac{\partial \sqrt{g} R}{\partial g_{k}^{\mu \nu}}+\partial_{k} \partial_{\ell} \frac{\partial \sqrt{g} R}{\partial g_{k \ell}^{\mu \nu}} .
$$


Then Eq.(10) takes the form

$$
[\sqrt{g} R]_{\mu \nu}=-\frac{\partial \sqrt{g} L}{\partial g^{\mu \nu}} .
$$

Note that in Hilbert's method of the gravitation equations derivation one does not need concrete specification for the Lagrangean function of the material system. In paper [6] D. Hilbert infers, in Theorem II, the identity:

$$
\delta_{L}(\sqrt{g} J)+\partial_{\lambda}\left(\delta x^{\lambda} \sqrt{g} J\right)=0
$$

where $\delta_{L}$ is the Lie derivative; $J$ is an arbitrary function invariant under coordinate transformations. He uses this identity when obtaining Eq.(48).

Then D. Hilbert proves a very important theorem III: "Let $J$ is an invariant depending only on the components of $g^{\mu \nu}$ and their derivatives; the variational derivatives of $\sqrt{g} J$ in $g^{\mu \nu}$ are designated, as earlier, as $[\sqrt{g} J]_{\mu \nu}$. If $h^{\mu \nu}$ is an arbitrary contravariant tensor then the quantity

$$
\frac{1}{\sqrt{g}}[\sqrt{g} J]_{\mu \nu} h^{\mu \nu}
$$

is also invariant; if one substitutes the standard tensor $p^{\mu \nu}$ instead of $h^{\mu \nu}$ and writes

$$
[\sqrt{g} J]_{\mu \nu} p^{\mu \nu}=\left(i_{s} p^{s}+i_{s}^{e} p_{e}^{s}\right)
$$

where expressions

$$
\begin{aligned}
& i_{s}=[\sqrt{g} J]_{\mu \nu} \partial_{s} g^{\mu \nu}, \\
& i_{s}^{\ell}=-2[\sqrt{g} J]_{\mu s} g^{\mu \ell}
\end{aligned}
$$

depend only on $g^{\mu \nu}$ and their derivatives, then

$$
i_{s}=\frac{\partial i_{s}^{\ell}}{\partial x^{\ell}}
$$

in the sense that this equation holds identically for all arguments, i.e. $g^{\mu \nu}$ and their derivatives".

Hilbert applies this theorem to the case $J=R$. Then identity (17) assumes the form:

$$
\partial_{\ell}\left\{[\sqrt{g} R]_{s}^{\ell}\right\}+\frac{1}{2}[\sqrt{g} R]_{\mu \nu} \frac{\partial g^{\mu \nu}}{\partial x^{s}} \equiv 0
$$


This identity is similar to (3), hence one can write it in the form (4)

$$
\nabla_{\ell}[\sqrt{g} R]_{s}^{\ell} \equiv 0 .
$$

We see that the covariant derivative of the variational derivative $[\sqrt{g} R]_{s}^{\ell}$ is equal to zero. Thus, on the basis of (12) we get

$$
\nabla^{\ell}\left\{\frac{\partial \sqrt{g} L}{\partial g^{s \ell}}\right\}=0
$$

According to Hilbert the energy-momentum tensor density of the material system, $T_{\mu \nu}$, is defined as follows:

$$
T_{\mu \nu}=-\frac{\partial \sqrt{g} L}{\partial g^{\mu \nu}},
$$

and equality (20) can be written down as a covariant conservation law of the energy-momentum tensor of the material system:

$$
\nabla_{\nu} T_{\mu}^{\nu}=0 .
$$

It was Hilbert who gave for the first time the definition (21) of the energymomentum tensor of the material system and showed that this tensor satisfies Eq. (22); by that he gave a basis of the Einstein's assumption from Ref. [8]. So, D. Hilbert found the gravitational field equation ${ }^{3}$

$$
[\sqrt{g} R]_{\mu \nu}=-\varkappa T_{\mu \nu}
$$

from which the law of covariant conservation of energy-momentum (22) follows exactly.

Multiplying both parts of Eq. (23) by $g^{\mu \nu}$ and summing up in indices $\mu$ and $\nu$ we get

$$
g^{\mu \nu}[\sqrt{g} R]_{\mu \nu}=-\varkappa T .
$$

In the l.h.s. of Eq.(24) an invariant is formed, which contains second derivatives linearly. But there exists only one such invariant, $R$. One gets thereof the equation

$$
\sqrt{g} \beta R=-\varkappa T
$$

where $\beta$ is an arbitrary constant.

\footnotetext{
${ }^{3}$ Original paper [6] by Hilbert corresponds to the system of units where $\varkappa=1$. Authors
} 
Summarizing one can say that the gravitational field equations were found by D. Hilbert and, by that, the problem, posed by A. Einstein in 1913 was resolved. Eq. (23) are identical with Eq.(1). They differ only in the form. Below we will see that, according to Hilbert, Eqs. (23) are easily transformed into (1). Hilbert, both in the proofs and in paper [6], wrote: "In the following I want ...to establish . . a new system of fundamental equations of physics". And further: "...my fundamental equations", "...my theory. . ". D. Hilbert could not write so, if he did not considered himself the author of the "fundamental equations of physics".

The tensor density $[\sqrt{g} R]_{\mu \nu}$ in Eq. (23) contains by construction the second order derivatives linearly, so, on the basis of (10a) this energy density has the form

$$
[\sqrt{g} R]_{\mu \nu}=\sqrt{g}\left(R_{\mu \nu}+\alpha g_{\mu \nu} R\right) .
$$

Expression (26) was quite evident for Hilbert. Maybe for the authors of $[2,3,4]$ it is difficult to understand that, but this is their personal affair. For the l.h.s. of Eq. (24) one obtains, on the basis of (26),

$$
g^{\mu \nu}[\sqrt{g} R]_{\mu \nu}=\sqrt{g}(4 \alpha+1) R,
$$

which is in complete correspondence with (25). Namely about these general reasonings Hilbert wrote: ". . what is clear without calculations if to take into account that $R$ is the only invariant and $R_{\mu \nu}$ is the only (besides $g_{\mu \nu}$ ) secondorder tensor, which can be constructed from $g_{\mu \nu}$ and its first and second derivatives $g_{k}^{\mu \nu}, g_{k \ell}^{\mu \nu}$.

Authors of paper [2] (see also [3]) write in this connection "This argument is, however, untenable, because there are many other tensors of second rank and many other invariants that can be constructed from the Riemann tensor".

This statement of the authors of [2] has no relation to the exact Hilbert's argument because the authors of papers [2,3] overlooked the main thing: one argued on the construction of the gravitational equations containing derivatives of $g^{\mu \nu}$ of order not higher than two. Hilbert specially wrote about that in his paper [6]: "If only second order derivatives of the potentials $g^{\mu \nu}$ can enter the gravitational equations, then the function $H$ has to have the form

$$
H=R+L " .
$$


Therefore D. Hilbert was absolutely right that in this case only $R$ and two tensors, $R_{\mu \nu}$ and $g_{\mu \nu} R$, contain linearly second derivatives of the gravitational potential $g^{\mu \nu}$. All other tensors with such properties are linear combinations of these tensors.

Likewise the author of the paper $[4]$ is wrong when he writes: $\ll \ldots$ variational derivation of the equations is absent, and the right form of the equations (with the "half" term) is motivated (not quite correctly) by the uniqueness of the Ricci tensor and the scalar curvature as generally covariant quantities, depending only on $g^{\mu \nu}$ 's and their first and second derivatives $\gg$.

It is astonishing indeed when the author of the paper [4] writes about Hilbert's paper: "...variational derivation... is absent". He probably forgot a well known circumstance that the Lagrange equations, which were presented by Hilbert, are a consequence of the least action principle (Axiom I of Hilbert). Thus, the variational derivation of the gravitational field equation takes place in Hilbert's paper [6].

How the authors of $[2,3,4]$ make up their mind to analyze and to judge D. Hilbert's papers [6] if they do not understand the essence of his exact mathematical arrguments? The authors of papers [2,3] write further: "Even if one requires the tensors and invariants to be linear in the Riemann tensor, the crucial coefficient of the trace term remains undetermined by such an argument". This is again wrong, it is easily determined. Hilbert proved the identity (19)

$$
\nabla_{\sigma}[\sqrt{g} R]_{\mu}^{\sigma} \equiv 0 .
$$

With account of Eq. (26) and with use of the local frame where Christoffel's symbols are zero, the identity (28) takes a simple form:

$$
\partial_{\sigma}\left(R_{\mu}^{\sigma}+\alpha \delta_{\mu}^{\sigma} R\right) \equiv 0 .
$$

From (8) and (9) one finds

$$
\partial_{\mu} R=K_{\mu}, \quad \partial_{\sigma} R_{\mu}^{\sigma}=\frac{1}{2} K_{\mu},
$$

where

$$
K_{\mu}=g^{\nu \sigma} g^{\lambda \rho} \partial_{\sigma} \partial_{\nu} \partial_{\mu} g_{\lambda \rho}-g^{\nu \sigma} g^{\alpha \lambda} \partial_{\sigma} \partial_{\alpha} \partial_{\mu} g_{\lambda \nu} .
$$

Making use of these expressions we get

$$
\partial_{\sigma}\left(R_{\mu}^{\sigma}+\alpha \delta_{\mu}^{\sigma} R\right)=\left(\frac{1}{2}+\alpha\right) K_{\mu} \equiv 0 .
$$


We have thereof

$$
\alpha=-\frac{1}{2}
$$

and hence,

$$
[\sqrt{g} R]_{\mu \nu}=\sqrt{g}\left(R_{\mu \nu}-\frac{1}{2} g_{\mu \nu} R\right)
$$

i.e.

$$
\sqrt{g}\left(R_{\mu \nu}-\frac{1}{2} g_{\mu \nu} R\right)=-\varkappa T_{\mu \nu} .
$$

Thus "the critical coefficient", that is a concern of the authors of $[2,3]$, is obtained in Hilbert's approach in a trivial way by taking derivatives fairly accessible to a first-year student of a university. It is also clear that the trace term $\frac{1}{2} g_{\mu \nu} R$ does not arise as a result of some arbitrary "introduction" into the field equations formulated by Hilbert; it is organically contained there.

Later, in 1921, in paper [10], A. Einstein would construct the geometrical part of the gravitational equations making use of the tensor

$$
R_{\mu \nu}+a g_{\mu \nu} R
$$

i.e. in the same way as it was done earlier by Hilbert at the transformation of gravitation equations (12) to the form (34). Creative endeavour of the authors of $[2,3]$ is crowned with the following thoughtful conclusion: "Taken together, this sequence suggests that knowledge of Einstein's result may have been crucial to Hilbert's introduction of the trace term into his field equations".

How can one arrive to such an idea after reading Hilbert's paper? Let us remind to the authors of [2] that, in Hilbert's formalism, one does not need to introduce anything. As soon as one wrote the world function $H$ in the form

$$
\boldsymbol{H}=\boldsymbol{R}+\boldsymbol{L}
$$

and established Theorem III, the rest was just a matter of calculational techniques, and nothing more.

Thus, the analysis that we have undertaken on the judgements of the authors of [2] shows that all their reproofs to Hilbert are either wrong or do not concern him. So all their arguments in favour of the point of view "that radically differs" from the standard one are inconclusive. 
Hilbert certainly obtained, before publication of Einstein's paper with the trace term, the equality (33). Taking use of (19) and (33) we find

$$
\nabla_{\nu}\left(R_{\mu}^{\nu}-\frac{1}{2} \delta_{\mu}^{\nu} R\right) \equiv 0 .
$$

But this is the Bianchi identity.

Poor knowledge of Hilbert's paper can be met not only in Refs.[2]. For instance, A. Pais in the book [11], §15.3, wrote: "Evidently Hilbert did not know the Bianchi identities either!" and further: "I repeat one last time that neither Hilbert nor Einstein was aware of the Bianchi identities in that crucial November 1915". "Interesting enough, in 1917 the experts were not aware that Weyl's derivation of Eq. 15.4 (The identity in question. -Authors) by variational techniques was a brand new method for obtaining a long-known result". A. Pais was right in that A.Einstein did not know the Bianchi identity in that crucial November 1915. All the rest in [11], concerning Hilbert, is wrong. The matter is that Hilbert did not know the Bianchi identity, indeed. He just himself obtained it. D. Hilbert proved with variational method the general identity (see Theorem III by Hilbert), from which, putting $J=R$, he obtained also the Bianchi identity.

Thus it was not Weyl in 1917 but Hilbert in 1915 who obtained the Bianchi identity with variational method. A. Pais wrote in $\S 15.3$ "In November 1915, neither Hilbert nor Einstein was aware of this royal road to the conservation laws. Hilbert had come close".

The authors of [3] write similarly: “..... Hilbert did not discover royal road to the formulation of the field equations of general relativity. In fact, he did not formulate these equations at all...".

All this is wrong. Namely Hilbert found the shortest and general way to formulate the gravitational equations. He found the Lagrange function of the gravitational field, $R$, with help of which the gravitational equations are obtained automatically via the principle of the least action. One obtains them namely in such a way when giving an account of Einstein's General Relativity. It is a pity that A. Pais seems to look through the Hilbert paper superficially; the same is true for the authors of Refs. [2,3].

Later, in 1924, D. Hilbert wrote [12]: "In order to define the expression $[\sqrt{g} R]_{\mu \nu}$ one chooses first the frame in such a way that all $g_{s}^{\mu \nu}$, taken in the world point, disappear. We find thereof

$$
[\sqrt{g} R]_{\mu \nu}=\sqrt{g}\left[R_{\mu \nu}-\frac{1}{2} g_{\mu \nu} R\right] "
$$


Authors of [2] write, concerning this: "To summarize: Initially Hilbert did not give the explicit form of the field equations; then, after Einstein had published his field equations, Hilbert claimed that no calculation is necessary; finally, he conseded that one is.".

This statement is a creation of the mind of the authors of [2]. No solid reasons exist that Hilbert did not obtained, himself, the explicit form of the field equations. One obtains them in an elementary way from Eqs.(23) and expression (26) with the use of the identity (28). If one can seriously assume that Hilbert was not able to obtain (33) from (28)? Hilbert's addition made in 1924 does not mean a "recognition that calculation is necessary". He introduced it just to remind a simple method to find a tensor. This did not discard his exact argument ("...clear without calculation") at all.

The authors of $[2,3]$ claim, referring to the Proofs, that Hilbert had the gravitation equation only in the form (23). Equation (23) contains the derivatives

$$
\frac{\partial \sqrt{g} R}{\partial g^{\mu \nu}}, \quad \frac{\partial \sqrt{g} R}{\partial g_{k}^{\mu \nu}}, \quad \frac{\partial \sqrt{g} R}{\partial g_{k \ell}^{\mu \nu}} .
$$

It is impossible to imagine a physicist-theorist or mathematician who would not calculate these derivatives and obtain explicitly the differential equations containing only derivatives $g_{k}^{\mu \nu}, g_{k \ell}^{\mu \nu}$. As we have seen, it was not nesessary, for Hilbert, to calculate them, because he managed to identify the structure of the expression $[\sqrt{g} R]_{\mu \nu}$ from the general and rigorous mathematical statements, due to which the calculation of the "critial coefficient" became trivial.

That is why the conclusion of the authors of papers $[2,3,4]$, that Hilbert did not obtain the "explicit form of the gravitational field equations" cannot be true. It contradicts also, as we will see further, to the correspondence between Einstein and Hilbert, from which everything becomes absolutely clear, and no additional arguments are needed. There does not exist more decisive argument than the evidence of Einstein himself. But precisely this most important evidence of Einstein was left afield by the authors of $[2,3]$, who put into the center of their analysis unpublished and mutialted materials of Hilbert.

The Einstein evidence in his letter to Hilbert of 18 November 1915 excludes unambiguously any false conjectures about Hilbert's paper [6]. Thus, the "archive finding" of the authors of [2], as a matter of principle, cannot shatter the evidence of Einstein himself. One could stop here the further dis- 
cussion of the question. But the authors of [2,3,4] alongside their arguments make erroneous conclusions about Hilbert's paper [6]. So we would like to specially concentrate on this.

Even if one does not follow the general statements of Hilbert, it us still possible, making use of definition (11), to execute simple differentiation and to express the tensor density $[\sqrt{g} R]_{\mu \nu}$ in terms of the Ricci tensor density and scalar density $\sqrt{g} R$. The first term in (11) can be written in the form

$$
\frac{\partial \sqrt{g} R}{\partial g^{\mu \nu}}=\sqrt{g}\left(R_{\mu \nu}+\frac{1}{\sqrt{g}} \frac{\partial \sqrt{g}}{\partial g^{\mu \nu}} R\right)+\sqrt{g} g^{\alpha \beta} \frac{\partial R_{\alpha \beta}}{\partial g^{\mu \nu}},
$$

Because of

$$
\frac{\partial \sqrt{g}}{\partial g^{\mu \nu}}=-\frac{1}{2} \sqrt{g} g_{\mu \nu}
$$

we get

$$
\frac{\partial \sqrt{g} R}{\partial g^{\mu \nu}}=\sqrt{g}\left(R_{\mu \nu}-\frac{1}{2} g_{\mu \nu} R\right)+\sqrt{g} g^{\alpha \beta} \frac{\partial R_{\alpha \beta}}{\partial g^{\mu \nu}} .
$$

We have on the basis of (11) and (40):

$$
[\sqrt{g} R]_{\mu \nu}=\sqrt{g}\left(R_{\mu \nu}-\frac{1}{2} g_{\mu \nu} R\right)+\left\{\sqrt{g} g^{\alpha \beta} \frac{\partial R_{\alpha \beta}}{\partial g^{\mu \nu}}-\partial_{k} \frac{\partial \sqrt{g} R}{\partial g_{k}^{\mu \nu}}+\partial_{k} \partial_{\ell} \frac{\partial \sqrt{g} R}{\partial g_{k \ell}^{\mu \nu}}\right\} .
$$

It is easy to see that terms in figure parenthesis dissapear identically. The most simple way is to use the local Riemannian frame where Christoffel symbols are zero. In such a simple, but not very elegant, way we arrive again to the expression

$$
[\sqrt{g} R]_{\mu \nu}=\sqrt{g}\left(R_{\mu \nu}-\frac{1}{2} g_{\mu \nu} R\right) .
$$

The authors of paper [3] wrote: "In both the Proofs and the published version of paper [6], Hilbert erroneously claimed that one can consider the last four equations (i.e. electromagnetic field equations. - Authors) as a consequence of the 4 identities that must hold, according to his Theorem I, between the 14 differential equations...".

Things, however, are not such as the authors of [3] suppose. Theorems I and II are formulated for $J$, an invariant under arbitrary transformations of the four world parameters. According to these theorems, there exist four identities for any invariant. Hilbert, in his paper, considers two invariants, $R$ and $L$. The general invariant $H$ is composed of these two invariants:

$$
H=R+L \text {. }
$$


The gravitation equations, in Hilbert's notations, have the form:

$$
[\sqrt{g} R]_{\mu \nu}=-\varkappa T_{\mu \nu}
$$

Hilbert chooses the invariant $L$ as a function of the variables $g^{\mu \nu}, q_{\sigma}, \partial_{\nu} q_{\sigma}$ and so he obtains the generalized Maxwell equations

$$
[\sqrt{g} L]^{\nu}=0
$$

where

$$
[\sqrt{g} L]^{\nu}=\frac{\partial \sqrt{g} L}{\partial q_{\nu}}-\partial_{\mu}\left(\frac{\partial \sqrt{g} L}{\partial\left(\partial_{\mu} q_{\nu}\right)}\right) .
$$

Then, on the basis of Theorem II, Hilbert obtains that the Lagrange function $L$ depends on the derivatives of the potential $q_{\nu}$ only via the combination $F_{\mu \nu}$, i.e.

$$
L\left(F_{\mu \nu}\right)
$$

where

$$
F_{\mu \nu}=\partial_{\mu} q_{\nu}-\partial_{\nu} q_{\mu}
$$

On this basic Hilbert chooses the Lagrangean in the form

$$
L=\alpha Q+f(q)
$$

where

$$
Q=F_{\mu \nu} F_{\lambda \sigma} g^{\mu \sigma} g^{\nu \lambda}, \quad q=q_{\mu} q_{\nu} g^{\mu \nu},
$$

here $\alpha$ is a constant.

Hilbert then remarks that the equations of electrodynamics "can be considered as a consequence of the equations of gravitation".

According to Theorem II the four identities take place for the invariant $L$ :

$$
\nabla_{\mu} T_{\nu}^{\mu}=F_{\mu \nu}[\sqrt{g} L]^{\mu}+q_{\nu} \partial_{\mu}[\sqrt{g} L]^{\mu} .
$$

It follows from identity (47) that, if the equations of motion of a material system (41) hold, then the covariant conservation law takes place:

$$
\nabla_{\mu} T_{\nu}^{\mu}=0
$$

If one makes use of the gravitation equations (34) for identity (47) then Hilbert's equations result:

$$
F_{\mu \nu}[\sqrt{g} L]^{\mu}+q_{\nu} \partial_{\mu}[\sqrt{g} L]^{\mu}=0
$$


which were denoted in his paper [6] under the number (27).

Equations (48) have to be compatible with the equations, which follow from the principle of the least action with the same Lagrangean $\boldsymbol{L}$. It is only possible in the case, when the "generalized Maxwell equations" hold:

$$
[\sqrt{g} L]^{\nu}=0 .
$$

Therefore, the author of paper [4] is completely wrong, considering that "in the case of gauge-noninvariant Mie's theory with a Lagrangean of the kind (45) one has in general use not the generalized Maxwell equations (49), but rather equations (48)."

This statement contradicts the principle of the least-action, i.e. Hilbert's Axiom I. So, the four identities (47) due to Theorem II and equations of gravitation (34) lead to the four equations (48) which are compatible with the generalized Maxwell equations, obtained on the basis of Hilbert's Axiom I. This is what Hilbert emphasized in paper [6]. In this relation he pointed out: "... from the gravitation equations (10) there really follow 4 mutually independent linear combinations (48) of equations of the electrodynamics (41) (emphasized by the authors) altogether with their first derivatives".

One has to specially stress that Hilbert writes about "linear combinations of the equations of electrodynamics (41)", but not the expressions (42). Namely here the authors of [3,4] admit a confusion.

Let us note that in the particular case, when

$$
L=\alpha Q,
$$

the second term in Eq.(48) dissapears identically and we come to the equations

$$
F_{\mu \nu}[\sqrt{g} L]^{\mu}=0 .
$$

It follows therefore that if the determinant $\left|F_{\mu \nu}\right|$ is not zero, the Maxwell equations take place

$$
[\sqrt{g} L]^{\mu}=0,
$$

which are in full agreement with the principle of the least action (Hilbert's Axiom I). In such a way the Maxwell equation are the consequence of the gravitation equation (34) and four identities (47). All this follows from Hilbert's article if one reads it attentively. Afterwards Einstein 
together with Infeld and Hoffmann in [13], and also Fock in [14] would obtain the equation of motion of a material system from the gravitation equations.

One notices quite often that Hilbert obtained the gravitational field equation "... not for an arbitrary material system, but especially basing on Mie's theory" [15]. That is not quite right. Method which Hilbert used is general and no limitations are implied on the form of the function $L$.

The circumstance that the gravitation equations imply four equations for the material system, looked attractive for Hilbert and he applied his general equations to Mie's theory. Such a unification of gravitation and Mie's theory was not fruitful, but Hilbert's general method for obtaining the gravitation equations proved to be very far-reaching.

Now a few words about auxialiary noncovariant equations.

To solve a problem it is always necessary to have a complete system of equations. There are only ten equations of general relativity. One still needs to add four equations, which cannot be chosen generally covariant. These auxiliary conditions are called coordinate conditions, and can be of various kinds. Hilbert meant namely this when he wrote (see Proofs in [7]): "As our mathematical Theorem shows us, the previous Axioms I and II can give only 10 mutually independent equations for 14 potentials. On the other hand, due to general invariance, more than 10 essentially independent equations for 14 potentials, $g_{\mu \nu}, q_{s}$, are impossible, and, as we wish to hold on Cauchy's theory for differential equations and to give to basic equations of physics a definite character, an addition to (4) and (5) of auxiliary non-invariant equations is inevitable."

This is a mathematical requirement and it is necessary for a theory. Hilbert tried to obtain these additional equations in the framework of the very theory, but he failed to do this and did not include that into the published article.

So the basic system of the 10 equations of general relativity is generally covariant, but the complete system of equations which is necessary to solve problems is not generally covariant because four equations expressing coordinate restrictions cannot be tensorial; they are not generally covariant. The solution to a complete system of the gravitational field equations can be always written in any admissible coordinate system. Namely here a notion of the chart atlas arises.

That is why the statement of the authors of $[2,3,4]$ that Hilbert's theory is not generally covariant, in contrast with Einstein's theory, is wrong. The complete system of equations both of Hilbert and 


\section{Einstein is not generally covariant.}

The only difference was in that Hilbert tried to uniquely construct these non-covariant equations in the framework of the very theory. This appeared impossible. The equations defining the choice of frame became quite arbitrary but not tensorial.

In this relation J. Synge [16] writes: "One can find in the papers on general relativity a number of various coordinate conditions, pursuing every time special aims. In order to approach the problem in a unified way let us write down the coordinate conditions in the form

$$
C_{i}=0, \quad(i=0,1,2,3) .
$$

The metric tensor $g_{i j}$ must satisfy these equations (perhaps differential). Certainly, they cannot be tensorial, because they are satisfied only at a special choice of coordinates. ". What is the material on which the authors of paper [2] made their conclusions? In the so-called proofs of D. Hilbert's paper, they proceeded from, the invariants $H$ and $K$ are used but there is no their definition. D. Hilbert writes in the Proofs: "I would like to construct below a new system of basic equations of physics, following the axiomatic method and proceeding, essentially, from the three axioms".

Evidently, Hilbert had to define the invariants $H$ and $K$ in order to do that. It is impossible to imagine that Hilbert, having posed such an aim, did not define these fundamental quantities. But this means that the parts absent from the Proofs are very essential and contain an important information. Valid conclusions cannot be made without account of this key infromation.

However the authors of [2] neglected this important circumstance and were in a hurry to conclude that Hilbert did not derive the gravitation equations in the form

$$
\sqrt{g}\left(R_{\mu \nu}-\frac{1}{2} g_{\mu \nu} R\right)=-\varkappa T_{\mu \nu} .
$$

They presented this conclusion to the wide scientific community in a popular and well-known journal "Science" [2]. For all that the authors of [2] did not inform the readers that so-called Proofs are mutilated. Only later, in [3], they mentioned that. The authors of [2] claim that the Proofs allowed them to base their point of view "that radically differs from the standard" one. How could it be done on the basis of a preliminary and mutilated material?

Here is one more method of "analysis" used by the authors of [3]: "Remarkably, in characterizing his system of equations, Hilbert deleted the word 
"neu", a clear indication that he had meanwhile seen Einstein's paper and recognized that the equations implied by his own variational principle are formally equivalent to those which Einstein had explicitly written down (because of where the trace term occurs), if Hilbert's stress-energy tensor is substituted for the unspecified one on the right-hand side of Einstein field equations."

But all cited above loses sense because actually their "clear indication" disappears as D. Hilbert in the published article [6] wrote quite clearly: "I would like to construct below. . a new system of fundamental equations".

It is extremely tactless to produce conclusions on Hilbert's ideas on the basis of his marginal remarks in preliminary unpublished materials. The system of gravitational equations obtained by Hilbert is really the new one. He obtained it without knowledge that A. Einstein came to the same gravitational equations. That is what A. Einstein wrote to D. Hilbert about in the letter of 18 November 1915 (see Section 3). Strange is the way, chosen by the authors of [3], to base their "radically different" point of view. Many-page composition [3] abounds in both similar doubtful arguments and erroneous statements. Such an approach to the study of most important physics papers can be hardly considered as a professional, based on a profound analysis of the material.

In conclusion of this section let us note, that Hilbert's papers under general title “Grundlagen der Physik" are very important and instructive. It would be very good if theoreticians, who deal with similar problems, knew them.

Thus, for instance, an article [17] was published in "Uspekhi". Should the authors of this paper read Hilbert's paper [18], published in 1917, they would see that the critical coordinate velocity $V_{c}$, which they calculated approximately, is equal in fact to

$$
V_{c}=\frac{1}{\sqrt{3}}\left(\frac{r-\alpha}{r}\right), \quad \alpha=r_{g}=2 G M .
$$

Namely at this velocity the acceleration is equal to zero. Velocity $V_{c}$ depends on the radius, while the corresponding proper velocity, $v$, does not depend on $r$ and

$$
v=\frac{1}{\sqrt{3}}
$$

In order to obtain the critical coordinate velocity $V_{c}$ in the first order in $G$ one needs to keep in acceleration terms of the second order in $G$. Gravitational 
field does not exert an action on a body, if the latter moves with velocity $V_{c}$, under the action of some external force.

In paper [18] D. Hilbert obtains the equation

$$
\frac{d^{2} r}{d t^{2}}-\frac{3 \alpha}{2 r(r-\alpha)}\left(\frac{d r}{d t}\right)^{2}+\frac{\alpha(r-\alpha)}{2 r^{3}}=0
$$

and adduces its integral:

$$
\left(\frac{d r}{d t}\right)^{2}=\left(\frac{r-\alpha}{r}\right)^{2}+A\left(\frac{r-\alpha}{r}\right)^{3},
$$

where $A$ is a constant; for the light $A=0$.

One obtains thereof the formula (20) for the velocity from the paper [17]

$$
\left(\frac{d r}{d t}\right)^{2}=\frac{1}{3}\left(1-\frac{r_{g}}{r}\right)^{2}\left(1+\frac{2 r_{g}}{r}\right),
$$

which differs from the critical velocity $V_{c}$. At this velocity the acceleration is not zero.

D. Hilbert writes further: "According to this equation the acceleration is negative or positive, i.e. gravitation attracts or repulses dependent on if the absolute value of the velocity obeys to inequality

$$
\left|\frac{d r}{d t}\right|<\frac{1}{\sqrt{3}}\left(\frac{r-\alpha}{r}\right)
$$

or inequality

$$
\left|\frac{d r}{d t}\right|>\frac{1}{\sqrt{3}}\left(\frac{r-\alpha}{r}\right) " .
$$

For the light Hilbert finds

$$
\left|\frac{d r}{d t}\right|=\frac{r-\alpha}{r},
$$

and further he notes: "The light propagating rectilinearly towards the center experiences always a repulsion according to the latter inequalities; its speed increases from 0 at $r=\alpha$ to 1 at $r=\infty "$.

Let us note that the local speed of light is equal to 1 (in units of $c$ ). It is also necessary to note that the velocity $V_{c}$ is not a solution of the initial equation. 
One more remark. The authors of [17] write: «Maybe this is the reason why sometimes in the literature the proper time is called "genuine", or "physical". A lightminded person would think that any other time (the coordinate time) is not physical, and thus should not be considered》.

And further: "As a result some many specialists on general relativity consider coordinate-dependent quantities as nonphysical, so to say "secondquality" quantities. However the coordinate time is even more important for some problems than the proper time $\tau$ ".

So, as the authors of [17] notice: $\ll \ldots$ to speak about the proper time as a "genuine" or "physical" in contrast with the coordinate velocity is not logical». In vain the authors of [17] think that specialists in general relativity do not understand significance of coordinate quantities. All the description in general relativity proceeds in terms of coordinate quantities. One cannot avoid them in principle. This is well known for a long time.

As an example of the physical quantity let us take the proper time, which differs from the coordinate one in that it does not depend on the choice of the coordinate time. As one sees there is a difference, and it is quite essential. Another example is the coordinate velocity of light

$$
V=\frac{\sqrt{g_{00}}}{1-\frac{g_{0 i} e^{i}}{\sqrt{g_{00}}}},
$$

here $i=1,2,3 ; e^{i}$ is a unit vector in the three-dimensional Riemannian space.

The coordinate velocity $V$ is, certainly, measurable but depends on the choice of coordinates and can have an arbitrary value:

$$
0<V<\infty
$$

while the physical speed of light is equal exactly to $1(c)$. As one can see there is also a difference, and also very essential.

Therefore there is nothing "non-logical" in the use of notions of physical and coordinate velocities, contrary to the authors of [17].

\section{A. Einstein's approach}

Einstein wrote in 1913 [8]: "The theory stated in the following arose from the conviction that proportionality between the inertial and gravitational masses 
of bodies is an exact, real law of Nature, which must find its expression already in the basis of theoretical physics. Already in some earlier works I tried to express this conviction reducing the gravitational mass to the inertial one; this aspiration led me to the hypothesis that the gravity field (homogeneous in an infinitesimally small volume) can be physically substituted by an accelerated frame".

Namely this path led Einstein to the conviction that in general case the gravitational field is characterized by the ten space-time functions (metric coefficient of the Riemann space) $g_{\mu \nu}$

$$
d s^{2}=g_{\mu \nu}(x) d x^{\mu} d x^{\nu} .
$$

He further published a series of papers about which he wrote later in the paper [19]: «My efforts in recent years were directed toward basing a general theory of relativity, also for nonuniform motion, upon the supposition of relativity. I believed indeed to have found the only law of gravitation that complies with a reasonably formulated postulate of general relativity; and I tried to demonstrate the truth of precisely this solution in a paper ${ }^{4}$ [8] that appeared last year in the "Sitzungsberichte".

Renewed criticism showed to me that this truth is absolutely impossible to show in the manner suggested. That this seemed to be the case was based upon a misjudgment. The postulate of relativity - as far as I demanded it there - is always satisfied if the Hamiltonian principle is chosen as a basis. But in reality, it provides no tool to establish the Hamiltonian function $H$ of the gravitational field. Indeed, equation (77)l.c. which limits the choise of $H$ says only that $H$ has to be an invariant toward linear transformations, a demand that has nothing to do with the relativity of accelerations. Furthermore, the choice determine by equations (78)l.c. does not determine equation (77) in any way.

For these reasons I lost trust in the field equations I had derived, and instead looked for a way to limit the possibilities in a natural manner. In this pursuit I arrived at the demand of general covariance, a demand from which I parted, though with a heavy heart, three years ago when I worked together with my friend Grossmann. As a matter of fact, we were then quite close to that solution of the problem, which will be given in the following.

Just as the special theory of relativity is based upon postulate that all equations have to be covariant relative to linear orthogonal transformations,

\footnotetext{
${ }^{4}$ Equations of this paper are quoted in the following with the additional note "l.c." in order to keep them distinct from those in the present paper.
} 
so the theory developed here rests upon the postulate of the covariance of all systems of equations relative to transformations with the substitution determinant 1.

Nobody who really grasped it can escape from its charm, because it signifies a real triumph of the general differential calculus as founded by Gauss, Riemann, Christoffel, Ricci, and Levi-Civita. 》

Einstein chose the gravitation equation in the coordinate system $\sqrt{-g}=1$ in the form ${ }^{5}$

$$
\partial_{\alpha} \Gamma_{\mu \nu}^{\alpha}+\Gamma_{\mu \beta}^{\alpha} \Gamma_{\nu \text { alpha }}^{\beta}=-\varkappa T_{\mu \nu}
$$

where

$$
\Gamma_{\mu \nu}^{\alpha}=-\frac{1}{2} g^{\alpha \sigma}\left(\partial_{\mu} g_{\nu \sigma}+\partial_{\nu} g_{\mu \sigma}-\partial_{\sigma} g_{\mu \nu}\right),
$$

being $T_{\mu \nu}$ the energy-momentum tensor for a material system. The 1.h.s. of Eq.(52) is obtained from the Ricci tensor at the condition $\sqrt{-g}=1$.

Einstein finds the Lagrange function for the gravitational field

$$
L=g^{\sigma \tau} \Gamma_{\sigma \beta}^{\alpha} \Gamma_{\tau \alpha}^{\beta} .
$$

If one takes into account the relation

$$
2 \Gamma_{\sigma \beta}^{\alpha} \delta\left(g^{\sigma \tau} \Gamma_{\tau \alpha}^{\beta}\right)=\Gamma_{\sigma \beta}^{\alpha} \delta g_{\alpha}^{\sigma \beta}
$$

then it is easy to obtain:

$$
\delta L=-\Gamma_{\sigma \beta}^{\alpha} \Gamma_{\tau \alpha}^{\beta} \delta g^{\sigma \tau}+\Gamma_{\sigma \beta}^{\alpha} \delta g_{\alpha}^{\sigma \beta}
$$

We have thereof

$$
\frac{\partial L}{\partial g^{\mu \nu}}=-\Gamma_{\mu \beta}^{\alpha} \Gamma_{\nu \alpha}^{\beta}, \quad \frac{\partial L}{\partial g_{\alpha}^{\mu \nu}}=\Gamma_{\mu \nu}^{\alpha} .
$$

With help of these formula the gravitation equation (52) can be cast into the form

$$
\partial_{\alpha}\left(\frac{\partial L}{\partial g_{\alpha}^{\mu \nu}}\right)-\frac{\partial L}{\partial g^{\mu \nu}}=-\varkappa T_{\mu \nu} .
$$

Multiplying (57) by $g_{\sigma}^{\mu \nu}$ and summing up in indices $\mu$ and $\nu$, Einstein obtains

$$
\partial_{\lambda} t_{\sigma}^{\lambda}=\frac{1}{2} T_{\mu \nu} \partial_{\sigma} g^{\mu \nu}
$$

\footnotetext{
${ }^{5}$ In this Section we use Einstein's notations. (Authors)
} 
where the quantity

$$
t_{\sigma}^{\lambda}=\frac{1}{2 \varkappa}\left(\delta_{\sigma}^{\lambda} L-g_{\sigma}^{\mu \nu} \frac{\partial L}{\partial g_{\lambda}^{\mu \nu}}\right),
$$

characterizes the gravitational field. Taking into account the equality:

$$
\Gamma_{\mu \nu}^{\lambda} \partial_{\sigma} g^{\mu \nu}=2 g^{\alpha \mu} \Gamma_{\alpha \sigma}^{\nu} \Gamma_{\mu \nu}^{\lambda},
$$

one finds:

$$
t_{\sigma}^{\lambda}=\frac{1}{\varkappa}\left(\frac{1}{2} \delta_{\sigma}^{\lambda} g^{\mu \nu} \Gamma_{\mu \beta}^{\alpha} \Gamma_{\nu \alpha}^{\beta}-g^{\alpha \mu} \Gamma_{\alpha \sigma}^{\nu} \Gamma_{\mu \nu}^{\lambda}\right)
$$

All further calculations are made in the reference frame, where $\sqrt{-g}=1$. Einstein writes down the basic equations of gravitation (52) in the form

$$
\partial_{\alpha}\left(g^{\nu \lambda} \Gamma_{\sigma \nu}^{\alpha}\right)-\frac{1}{2} \delta_{\sigma}^{\lambda} g^{\mu \nu} \Gamma_{\mu \beta}^{\alpha} \Gamma_{\nu \alpha}^{\beta}=-\varkappa\left(T_{\sigma}^{\lambda}+t_{\sigma}^{\lambda}\right) .
$$

We will show below how close to the true gravitational field equations was Einstein when writing the paper of 4 November 1915 [19].

Since 1913 A. Einstein mentioned, in one or another way, that the quantity $\boldsymbol{t}_{\boldsymbol{\sigma}}^{\boldsymbol{\lambda}}$, characterizing the gravitational field must enter the gravitation equation in the same way as the quantity $\boldsymbol{t}_{\boldsymbol{\sigma}}^{\boldsymbol{\lambda}}$, characterizing material systems.

For instance, he wrote in 1913 in the paper [8]: “...the gravitational field tensor is a source of the field on equal foots with that of material systems, $\Theta_{\mu \nu}$. Exceptional position of the gravitational field energy in comparison with all other kinds of energy would lead to inadmissible consequences.". However, Einstein left aside this important intuituve argument when he wrote the paper [19].

In fact, the mentioned above consideration on a symmetry between the quantities $T_{\sigma}^{\lambda}$ and $t_{\sigma}^{\lambda}$ is rather a product of Einstein's intuition, but not a general physical principle. The matter is that the transformation properties of these quantities are different.

One has to notice that, as a rule, basic physical equations are not derived. Rather they are guessed on the basis of experimental data, general physical principles and intuition. That is why it is sometimes difficult to logically explain in what way they are obtained by an author. 
It is easy, with help of (60), to find the trace of the quantity $t_{\sigma}^{\lambda}$

$$
t=t_{\lambda}^{\lambda}=\frac{1}{\kappa} g^{\mu \nu} \Gamma_{\mu \beta}^{\alpha} \Gamma_{\nu \alpha}^{\beta}
$$

and to rewrite Einstein's equation (61) in the form

$$
\partial_{\alpha}\left(g^{\nu \lambda} \Gamma_{\sigma \nu}^{\alpha}\right)=-\varkappa\left(T_{\sigma}^{\lambda}+t_{\sigma}^{\lambda}-\frac{1}{2} \delta_{\sigma}^{\lambda} t\right) .
$$

It is seen that there is no symmetry between the quantities $T_{\sigma}^{\lambda}$ and $t_{\sigma}^{\lambda}$ in Eq. (63). One can easily see that this symmetry can be re-established in a simple way.

Consider first the conservation laws with help of (63). To this end we find the trace:

$$
\partial_{\alpha}\left(g^{\nu \beta} \Gamma_{\nu \beta}^{\alpha}\right)=-\varkappa(T-t)
$$

Now we multiply both parts of Eq.(64) by $\frac{1}{2} \delta_{\sigma}^{\lambda}$ and subtract the result from (63):

$$
\partial_{\alpha}\left(g^{\nu \lambda} \Gamma_{\sigma \nu}^{\alpha}-\frac{1}{2} \delta_{\sigma}^{\lambda} g^{\nu \beta} \Gamma_{\nu \beta}^{\alpha} \text { Bigr }\right)=-\varkappa\left(T_{\sigma}^{\lambda}+t_{\sigma}^{\lambda}-\frac{1}{2} \delta_{\sigma}^{\lambda} T\right) .
$$

One easily sees that the following equalities hold:

$$
\begin{gathered}
\partial_{\lambda} \partial_{\alpha}\left(g^{\nu \lambda} \Gamma_{\sigma \nu}^{\alpha}\right)=\frac{1}{2} \partial_{\lambda} \partial_{\alpha} \partial_{\sigma} g^{\alpha \lambda} \\
\partial_{\lambda} \partial_{\alpha} \delta_{\sigma}^{\lambda} g^{\nu \beta} \Gamma_{\nu \beta}^{\alpha}=\partial_{\lambda} \partial_{\alpha} \partial_{\sigma} g^{\alpha \lambda} .
\end{gathered}
$$

Making use of these equalities we find from Eq.(65):

$$
\partial_{\lambda}\left(T_{\sigma}^{\lambda}+t_{\sigma}^{\lambda}\right)=\frac{1}{2} \delta_{\sigma}^{\lambda} \partial_{\lambda} T
$$

similarly one can find, using (58), the relation

$$
\partial_{\lambda} T_{\sigma}^{\lambda}+\frac{1}{2} T_{\mu \nu} \partial_{\sigma} g^{\mu \nu}=\frac{1}{2} \delta_{\sigma}^{\lambda} \partial_{\lambda} T .
$$

It is evident from this that Eq. (63) does not provide the conservation laws, and also there is no symmetry between $T_{\sigma}^{\lambda}$ and $t_{\sigma}^{\lambda}$. To re-establish the symmetry in (63) and (68) it is necessary to make the folowing substitution:

$$
T_{\sigma}^{\lambda} \rightarrow T_{\sigma}^{\lambda}-\frac{1}{2} \delta_{\sigma}^{\lambda} T
$$


The trace of the tensor $T_{\mu \nu}$ is being changed as follows:

$$
T \rightarrow-T
$$

Note that symmetrization is not related to any assumptions on the structure of matter. Having completed this operation we obtain the new gravitational equations

$$
\partial_{\alpha}\left(g^{\nu \lambda} \Gamma_{\sigma \nu}^{\alpha}\right)=-\varkappa\left\{\left(T_{\sigma}^{\lambda}+t_{\sigma}^{\lambda}\right)-\frac{1}{2} \delta_{\sigma}^{\lambda}(T+t)\right\} .
$$

The same operation applied to (68) and (69) leads to re-establishing of the conservation laws

$$
\begin{gathered}
\partial_{\lambda}\left(T_{\sigma}^{\lambda}+t_{\sigma}^{\lambda}\right)=0 \\
\partial_{\lambda} T_{\sigma}^{\lambda}+\frac{1}{2} T_{\mu \nu} \partial_{\sigma} g^{\mu \nu}=0 .
\end{gathered}
$$

Eqs. (73) and (74) arise only from new equations (72).

In the supplement [20] to the article [19] Einstein makes a further step and chooses the gravitational equations in the form

$$
R_{\mu \nu}=-\varkappa T_{\mu \nu}
$$

generally covariant under arbitrary coordinate transformations. He abandons the condition $\sqrt{-g}=1$. In the frame $\sqrt{-g}=1$ these equations are equivalent to Eq.(52). But Eq.(52) does not provide neither the symmetry between $T_{\sigma}^{\lambda}$ and $t_{\sigma}^{\lambda}$ nor the conservation laws. So, it is natural to make the symmetrisation operations, (70) and (71), in the initial equations (75) as well. In such a way we obtain a new gravitation equation

$$
R_{\mu \nu}=-\varkappa\left(T_{\mu \nu}-\frac{1}{2} g_{\mu \nu} T\right)
$$

Namely these equations were obtained by Einstein several days later and published then in the paper [5]. Note that Einstein found the conservation law equations (73) still with the gravitation equations (63).

This sircumstance, probably, satisfied him, and he did not pay attention to a symmetry breaking between $T_{\sigma}^{\lambda}$ and $t_{\sigma}^{\lambda}$ in Eqs.(63).

However his method to satisfy the conservation laws led to the situation when the choice of the frame $\sqrt{-g}=1$ was possible only if the trace of the material tensor were put zero. Instead of re-establishing the symmetry 
via (70) and (71) Einstein chose another, more radical, way. He pushed forward a new physical idea [20], that "in reality only the quantity $T_{\mu}^{\mu}+$ $t_{\mu}^{\mu}$ is positive, while $T_{\mu}^{\mu}$ disappears". Such an approach re-established the symmetry. Nonetheless whatever radical, this approach was not fruitful, and this idea existed but short time.

Later Einstein returned to his old idea on symmetry and obtained in Ref. [5] the gravitational field equations (76). He mentioned there: "As it is not difficult to see, our additional term leads to that energy tensors of the gravitational field and of matter enter Eq.(9) in the same way".

There is some inexactitude in this statement. There does not exist, in general relativity, a gravitational field energy tensor.

Nonetheless, due to intuitive considerations, the use of such a quantity led Einstein directly to his goal.

We see that the way of Einstein led him inevitably to the same equations, which Hilbert obtained as well. It is quite evident that Einstein obtained them independently. Moreover, he gained them through much suffering during several years.

For better understanding of what is written above, of no small importance is quite a vivid correspondence [9] between Hilbert anf Einstein, which took place just in the period of their work on the gravitational field equations. Namely this correspondence witness that no "radically different" point of view, other than the standard one, can exist, as a matter of principle.

\section{Einstein-Hilbert Correspondence}

\section{From Einstein to Hilbert}

\section{Berlin, Sunday, 7 November 1915}

"Highly esteemed Colleague,

With return post I am sending you the correction to a paper in which I changed the gravitational equations, after having myself noticed about 4 weeks ago that my method of proof was a fallacious one. My colleague Sommerfeld wrote that you also have found a hair in my soup that has spoiled it entirely for you. I am curious whether you will take kindly to this new solution.

With cordial greetings, yours

A. Einstein 
When may I expect the mechanics and history week to take place in Göttingen? I am looking forward to it very much."

\section{From Einstein to Hilbert}

\section{Berlin, Friday, 12 November 1915}

"Highly esteemed Colleague,

I just thank you for the time being for your kind letter. The problem has meanwhile made new progress. Namely, it is possible to exact general covariance from the postulate $\sqrt{-g}=1$; Riemann's tensor then delivers the gravitation equations directly. If my present modification (which does not change the equations) is legitimate, then gravitation must play a fundamental role in the composition of matter. My own curiousity is interfering with my work! I am sending you two copies of last year's paper. I have only two other intact copies myself. If comeone else needs the paper, he can easily purchase one, of course, for $2 M$ (as an Academy offprint).

Cordial greetings, yours

Einstein"

\section{From Hilbert to Einstein}

\section{Göttingen, 13 November 1915}

"Dear Collegue,

Actually, I first wanted to think of a very palpable application for physicists, namely reliable relations between the physical constants, before obliging with my axiomatic solution to your great problem. But since you are so interested, I would like to lay out my theory in very complete detail on the coming Tuesday, that is, the day after the day after tomorrow (the 16th of this mo.). I find it ideally handsome mathematically and absolutely compelling according to axiomatic method, even to the extent that not quite transparent calculations do not occur at all and therefore rely on its factuality. As a result of gen. math. law, the (generalized Maxwellian) electrody. eqs. as a math. consequence of the gravitation eqs., such that gravitation and electrodynamics are actually nothing different at all. Furthermore, my energy concept forms the basis: $E=\sum\left(e_{s} t^{s}+e_{i h} t^{i h}\right)$, which is likewise a general invariant, and from this then also follow from a very simple axiom the 4 missing "space-time equations" $e_{s}=0$. I derived most pleasure in the discovery already duscussed with Sommerfeld that normal electrical energy results when a specific abso- 
lute invariant is differentiated from the gravitation potentials and then $g$ is set $=0.1$. My request is thus to come for Tuesday. You can arrive at 3 or 1/2 past 5. The Math. Soc. meets at 6 o'clock in the auditorium building. My wife and I would be very pleased if you stayed with us. It would be better still if you came already on Monday, since we have the phys. colloquium on Monday, 6 o'clock, at the phys. institute. With all good wishes and in the hope of soon meeting again, yours,

Hilbert

As far as I understand your new paper, the solution given by you is entirely different from mine, especially since my $e_{s}$ 's must also necessarily contain the electrical potential."

\section{From Einstein to Hilbert}

"Highly esteemed Colleague,

\section{Berlin, Monday, 15 November 1915}

Your analysis interests me tremendously, especially since I often racked my brains to construct a bridge between gravitation and electromagnetics. The hints your give in your postcards awaken the greatest of expectations. Nevertheless, I must refrain from travelling to Göttingen for the moment and rather must wait patiently untill I can study your system from the printed article; for I am tired out and plagued with stomach pains besides. If possible, please send me a correction proof of your study to mitigate my impatience.

With best regards and cordial thanks, also to Mrs. Hilbert, yours,

\section{A. Einstein"}

16 November 1915 D. Hilbert presented his result publicly. The author of the paper [21] writes about that:

« "Grundgleichungen der Physik" was the title of Hilbert's lecture to the Göttingen Mathematical Society of November 16. It was also the title under which his communication in the letter of invitation circulated among the Academy members between November 15 and the meeting of November $20 \ldots \gg$

He mentions also:

$\ll$ The invitation for the meeting of 20 November was issued on November 15 and was, as always, circulated among the members to confirm their participation and announce any communications they intended to present at the meeting. Into this invitation Hilbert wrote: "Hilbert legt vor in die Nachrichten: Grundgleichungen der Physik." 》 
"In response to Einstein's request", as the author of Ref.[21] notices, "Hilbert had to report his findings in correspondence to Einstein, unfortunately lost. He probably sent Einstein the manuscript of his lecture to the Götingen Mathematical Society, or a summary of its main points." 


\section{Einstein to Hilbert}

Berlin, 18 November, 1915

"Dear Colleague,

The system you furnish agrees — as far as I can see - exactly with what I found in the last few weeks and have presented to the Academy. The difficulty was not in finding generally covariant equations for the $g_{\mu \nu}$ 's; for this is easily achieved with the aid of Riemann's tensor. Rather, it was hard to recognize that these equations are a generalization, that is, simple and natural generalization of Newton's law. It has just been in the last few weeks that I succeeded in this (I sent you my communications), whereas 3 years ago with my friend Grossmann I had already taken into consideration the only possible generally covariant equations, which have now been shown to be the correct ones. We had only heavy-heartedly distanced ourselves from it, because it seemed to me that the physical discussion yielded an incongruency with Newton's law. The important thing is that the difficulties have now been overcome. Today I am presenting to the Academy a paper in which I derive quantitatively out of general relativity, without any guiding hypothesis, the perihelion motion of Mercury discovered by Le Verrier. No gravitation theory had achieved this untill now.

Best regards, yours

Einstein”

Such is the content of Einstein's reply letter. There does not exist an argument more forcible than the words in the letter, written by Einstein, himself: "The system you furnish agrees — as far as I can see - exactly with what I found in the last few weeks and have presented to the Academy". But namely this exact evidence remained aside in Refs. $[2,3,4]$. Already this only evidence by Einstein is fairly sufficient to exclude completely and forever any attempts to push forward "a point of view that radically differs from the standard point of view".

The authors of $[2,3]$ made a whole series of other wrong conclusions about Hilbert's paper. That is why we had to consider, in Section 1, their compositions in some detail.

Let us nonetheless assume that Einstein received from Hilbert the gravi- 
tation equations in the form (12), i.e.

$$
[\sqrt{g} R]_{\mu \nu}=-\frac{\partial \sqrt{g} L}{\partial g^{\mu \nu}}
$$

It is unbelievable that Einstein would consider that these equations agreed with his equations

$$
R_{\mu \nu}=-\varkappa\left(T_{\mu \nu}-\frac{1}{2} g_{\mu \nu} T\right)
$$

where the Ricci tensor enters explicitly. To agree that Eqs. (77) coincide with his equations (78) Einstein would need to calculate the derivatives

$$
\frac{\partial \sqrt{g} R}{\partial g^{\mu \nu}}, \quad \frac{\partial \sqrt{g} R}{\partial g_{k}^{\mu \nu}}, \quad \frac{\partial \sqrt{g} R}{\partial g_{k \ell}^{\mu \nu}} .
$$

However he did not calculate them that time. He wrote about that later, in the letter to H.A. Lorentz of 19 January 1916 [9]: "I avoided the somewhat involved computation of the $\partial R / \partial g^{\mu \nu}$ 's and $\partial R / \partial g_{\sigma}^{\mu \nu}$ 's by setting up the tensor equations directly. But the other way is certainly also workable and even more elegant mathematically".

It is also improbable that Hilbert, knowing that Ricci tensor enters the Einstein equations (he was informed of that in the letter from Einstein of 7 November 1915), could send him his equations in the form (77). No doubt that Einstein received from Hilbert the equations in the form

$$
\sqrt{g}\left(R_{\mu \nu}-\frac{1}{2} g_{\mu \nu} R\right)=-\frac{\partial \sqrt{g} L}{\partial g^{\mu \nu}},
$$

because it was not difficult for Hilbert, to find, from general considerations and practically without computations, as we have seen above, the equality

$$
[\sqrt{g} R]_{\mu \nu}=\sqrt{g}\left(R_{\mu \nu}-\frac{1}{2} g_{\mu \nu} R\right)
$$

In the letter to Hilbert of 18 November 1915 Einstein wrote: "The system you furmish agrees — as far as I can see - exactly to what I found...". It is easy to be persuaded in this if to compare Eqs.(78) and 
(79). Einstein's words "as far as I can see" were possibly caused by that in Hilbert's paper the energy-momentum tensor density was defined as

$$
\frac{\partial \sqrt{g} L}{\partial g^{\mu \nu}}
$$

where $L$ is a function of $g^{\mu \nu}, q_{\sigma}$ and $q_{\sigma \nu}$. Such a definition was new and unknown to Einstein. Time needed to understand its essence. But Einstein replied to Hilbert immediately. Later on, in the paper [22], Einstein would take advantage of namely such a definition of the energy-momentum tensor. He, in this paper, introduced, like Hilbert, a function $\mathcal{M}$ of variables $g^{\mu \nu}, q_{(\rho)}, q_{(\rho) \alpha}$ and wrote down the energy-momentum tensor density in the form

$$
\mathcal{T}_{\mu \nu}=-\frac{\partial \mathcal{M}}{\partial g^{\mu \nu}}
$$

Therefore it is impossible to understand on what ground the authors of [3] try to conclude quite an opposite: "The new energy expression that Hilbert now took over from Einstein ...". As we have just seen it is absolutely wrong. Namely Einstein adopted from Hilbert the definition of the energymomentum tensor density and used it in the paper [22].

Furthermore the authors of [3] conclude: “... Einstein's generalization of Hilbert's derivation made it possible to regard the latter as merely representing a problematic special case".

All this is wrong. Hilbert's method is general; it allows to obtain the gravitation equation without assumption on a concrete form of the Lagrange function $L$ of a material system. Therefore there was no (and could not be) generalization of the Hilbert inference. This is another story that afterwards Hilbert applied his method to the concrete case of Mie's theory.

As we have already mentioned in Section 1, the transformation of (77) to (79) was not a great labour for Hilbert with help of Theorem III, proven by him.

So the Proofs, moreover mutilated, cannot witness that Hilbert did not put the gravitational field equations in the form (79).

\section{Conclusion}

The analysis, undertaken in Sections 1 and 2, shows that Einstein and Hilbert inependently discovered the gravitational field equations. Their pathways 
were different but they led exactly to the same result. Nobody "nostrified" the other. So no "belated decision in the Einstein-Hilbert priority dispute", about which the authors of [2] wrote, can be taken. Moreover, the very Einstein-Hilbert dispute never took place.

All is absolutely clear: both authors made everything to immortalize their names in the title of the gravitational field equations.

But general relativity is Einstein's theory.

\section{Acknowledgement}

The authors are indebted to S.S. Gershtein and N.E. Tyurin for valuable discussions of the paper and to C.J. Bjerknes for helpful remarks.

\section{Appendix}

Below we shall give, with pedagogical purposes, the detailed proof of Hilbert's theorems II and III.

\section{Theorem II}

If $H$ is an invariant that depends on $g_{\mu \nu}, \partial_{\lambda} g_{\mu \nu}, \partial_{\sigma} \partial_{\lambda} g_{\mu \nu}, A_{\nu}$ and $\partial_{\lambda} A_{\nu}$, then for an infinitesimal contravariant vector $\delta x^{s}$ the following identity holds:

$$
\delta_{L}(\sqrt{g} H)=\partial_{s}\left(\sqrt{g} H \delta x^{s}\right) ;
$$

here $\delta_{L}$ is the Lie variation.

To prove this theorem consider the integral

$$
S=\int_{\Omega} d^{4} x \sqrt{g} H .
$$

Let us make an infinitesimal coordinate transformation

$$
x^{\prime \nu}=x^{\nu}+\delta x^{\nu} ;
$$

here $\delta x^{\nu}$ is an arbitrary infinitesimal four-vector. 
At this transformation the integral remains intact and theoref the variation $\delta_{c} S$ disappears:

$$
\delta_{c} S=\int_{\Omega^{\prime}} d^{4} x^{\prime} \sqrt{g^{\prime}} H^{\prime}-\int_{\Omega} d^{4} x \sqrt{g} H=0 .
$$

The first integral may be written as

$$
\int_{\Omega^{\prime}} d^{4} x^{\prime} \sqrt{g^{\prime}} H^{\prime}=\int_{\Omega} J \sqrt{g^{\prime}} H^{\prime} d^{4} x .
$$

Here $J$ is the Jacobian of the transformation

$$
J=\frac{\partial\left(x^{\prime 0}, x^{\prime 1}, x^{\prime 2}, x^{\prime 3}\right)}{\partial\left(x^{0}, x^{1}, x^{2}, x^{3}\right)} .
$$

Jacobian of the transformations $(A .3)$ is

$$
J=1+\partial_{\lambda} \delta x^{\lambda}
$$

Expanding $\sqrt{g^{\prime}} H^{\prime}$ into the Taylor series one finds

$$
\sqrt{g^{\prime}\left(x^{\prime}\right)} H^{\prime}\left(x^{\prime}\right)=\sqrt{g^{\prime}(x)} H^{\prime}(x)+\delta x^{\lambda} \partial_{\lambda}(\sqrt{g} H) .
$$

Due to $(A .5),(A .7)$ and $(A .8)$ equality $(A .4)$ assumes the form:

$$
\delta_{c} S=\int_{\Omega} d^{4} x\left[\delta_{L}(\sqrt{g} H)+\partial_{\lambda}\left(\sqrt{g} H \delta x^{\lambda}\right)\right]=0 .
$$

The Lie variation is

$$
\delta_{L}(\sqrt{g} H)=\sqrt{g^{\prime}(x)} H^{\prime}(x)-\sqrt{g(x)} H(x) .
$$

The Lie variation commutes with partial derivatives:

$$
\delta_{L} \partial_{\lambda}=\partial_{\lambda} \delta_{L}
$$

The Lie variation of $\sqrt{g} H$ is

$$
\delta_{L}(\sqrt{g} H)=P_{g}(\sqrt{g} H)+P_{q}(\sqrt{g} H),
$$


where

$$
\begin{gathered}
P_{g}(\sqrt{g} H)=\frac{\partial \sqrt{g} H}{\partial g_{\mu \nu}} \delta_{L} g_{\mu \nu}+\frac{\partial \sqrt{g} H}{\partial\left(\partial_{\lambda} g_{\mu \nu}\right)} \partial_{\lambda} \delta_{L} g_{\mu \nu}+\frac{\partial \sqrt{g} H}{\partial\left(\partial_{\sigma} \partial_{\lambda} g_{\mu \nu}\right)} \partial_{\sigma} \partial_{\lambda} \delta_{L} g_{\mu \nu}, \\
P_{q}(\sqrt{g} H)=\frac{\partial \sqrt{g} H}{\partial A_{\lambda}} \delta_{L} A_{\lambda}+\frac{\partial \sqrt{g} H}{\partial\left(\partial_{\sigma} A_{\lambda}\right)} \partial_{\sigma} \delta_{L} A_{\lambda} .
\end{gathered}
$$

Due to arbitrariness of the volume $\Omega$ one gets on the basis of $(A .9)$ the sesired Hilbert identity:

$$
\delta_{L}(\sqrt{g} H)+\partial_{\lambda}\left(\sqrt{g} H \delta x^{\lambda}\right) \equiv 0
$$

where

$$
\delta_{L}(\sqrt{g} H)=P_{g}(\sqrt{g} H)+P_{q}(\sqrt{g} H)
$$

\section{Theorem III}

If an invariant depends on $g_{\mu \nu}, \partial_{\lambda} g_{\mu \nu}, \partial_{\sigma} \partial_{\lambda} g_{\mu \nu}$ then the variational derivative

$$
\frac{\delta \sqrt{g} H}{\delta g_{\mu \nu}}=\sqrt{g} G^{\mu \nu}=\frac{\partial \sqrt{g} H}{\partial g_{\mu \nu}}-\partial_{\lambda} \frac{\partial \sqrt{g} H}{\partial\left(\partial_{\lambda} g_{\mu \nu}\right)}+\partial_{\sigma} \partial_{\lambda} \frac{\partial \sqrt{g} H}{\partial\left(\partial_{\sigma} \partial_{\lambda} g_{\mu \nu}\right)}
$$

satisfies the identity

$$
\nabla_{\lambda} G^{\lambda \nu} \equiv 0
$$

or, in another form,

$$
\partial_{\lambda}\left(\sqrt{g} G_{\rho}^{\lambda}\right)+\frac{1}{2} \sqrt{g} G_{\lambda \sigma} \partial_{\rho} g^{\lambda \sigma} \equiv 0,
$$

where $\nabla_{\lambda}$ stands for the covariant derivative in the Riemann space.

To prove this theorem consider the integral

$$
\int_{\Omega} \sqrt{g} H d^{4} x
$$

over a finite region of the four-dimensional world. The translation vector $\delta x^{\sigma}$ in $(A .3)$ has to disappear together with its derivatives on the 3-dimensional border of the region $\Omega$. This implies dissapearing of the field variations and their derivatives on the border of this region. Taking use of the Hilbert identity $(A .15)$ one finds:

$$
\int_{\Omega} \delta_{L}(\sqrt{g} H) d^{4} x=0
$$


In our case

$$
\delta_{L}(\sqrt{g} H)=P_{g}(\sqrt{g} H) .
$$

Expression $(A .13)$ can be written in the form

$$
P_{g}(\sqrt{g} H)=\frac{\delta \sqrt{g} H}{\delta g_{\mu \nu}} \delta_{L} g_{\mu \nu}+\partial_{\lambda} S^{\lambda},
$$

where vector $S^{\lambda}$ is

$$
S^{\lambda}=\left[\frac{\partial \sqrt{g} H}{\partial\left(\partial_{\lambda} g_{\mu \nu}\right)}-\partial_{\sigma}\left(\frac{\partial \sqrt{g} H}{\partial\left(\partial_{\sigma} \partial_{\lambda} g_{\mu \nu}\right)}\right)\right] \delta_{L} g_{\mu \nu}+\frac{\partial \sqrt{g} H}{\partial\left(\partial_{\sigma} \partial_{\lambda} g_{\mu \nu}\right)} \partial_{\sigma} \delta_{L} g_{\mu \nu} .
$$

Substituting (A.22) into (A.20) one finds:

$$
\int_{\Omega} \frac{\delta \sqrt{g} H}{\delta g_{\mu \nu}} \delta_{L} g_{\mu \nu} d^{4} x=0 .
$$

Now we shall find the variation $\delta_{L} g_{\mu \nu}$ at the transformations (A.3). Metric tensor $g_{\mu \nu}$ is transformed as

$$
g_{\mu \nu}^{\prime}\left(x^{\prime}\right)=\frac{\partial x^{\lambda}}{\partial x^{\prime \mu}} \cdot \frac{\partial x^{\sigma}}{\partial x^{\prime \nu}} g_{\lambda \sigma}(x) .
$$

One finds thereof for the transformation $(A .3)$

$$
\delta_{L} g_{\mu \nu}(x)=-\delta x^{\sigma} \partial_{\sigma} g_{\mu \nu}-g_{\mu \sigma} \partial_{\nu} \delta x^{\sigma}-g_{\nu \sigma} \partial_{\mu} \delta x^{\sigma} .
$$

With account of the equality

$$
\nabla_{\sigma} g_{\mu \nu}=\partial_{\sigma} g_{\mu \nu}-g_{\lambda \mu} \Gamma_{\sigma \nu}^{\lambda}-g_{\lambda \nu} \Gamma_{\sigma \mu}^{\lambda}=0,
$$

one can write the Lie derivative in the covariant form:

$$
\delta_{L} g_{\mu \nu}=-g_{\mu \sigma} \nabla_{\nu} \delta x^{\sigma}-g_{\nu \sigma} \nabla_{\mu} \delta x^{\sigma} .
$$

Substituting this expression into the integral (A.24) one gets

$$
\int_{\Omega} d^{4} x \frac{\delta \sqrt{g} H}{\delta g_{\mu \nu}} g_{\mu \sigma} \nabla_{\nu} \delta x^{\sigma}=0 .
$$


Eq. (A.28) can be written in the form

$$
\int_{\Omega}\left[\nabla_{\nu}\left(\frac{\delta \sqrt{g} H}{\delta g_{\mu \nu}} g_{\mu \sigma} \delta x^{\sigma}\right)-\delta x^{\sigma} \nabla_{\nu}\left(\frac{\delta \sqrt{g} H}{\delta g_{\mu \nu}} g_{\mu \sigma}\right)\right] d^{4} x=0 .
$$

Note, that

$$
\nabla_{\nu}\left(\frac{\delta \sqrt{g} H}{\delta g_{\mu \nu}} g_{\mu \sigma} \delta x^{\sigma}\right)=\partial_{\nu}\left(\frac{\delta \sqrt{g} H}{\delta g_{\mu \nu}} g_{\mu \sigma} \delta x^{\sigma}\right) .
$$

Due to $(A .30)$ the integral of the first term in the l.h.s. of $(A .29)$ disappears and Eq. (A.29) assumes the form:

$$
\int_{\Omega} \delta x^{\sigma} \nabla_{\nu} G_{\sigma}^{\nu} d^{4} x=0
$$

Here we have introduced in accordance with definition $(A .17)$ the mixed tensor:

$$
\sqrt{g} G_{\sigma}^{\nu}=\frac{\delta \sqrt{g} H}{\delta g_{\mu \nu}} g_{\mu \sigma} .
$$

We find, due to arbitrariness of the vector $\delta x^{\sigma}$ the desired Hilbert identity

$$
\nabla_{\nu} G_{\sigma}^{\nu} \equiv 0
$$

or, in more detail,

$$
\nabla_{\nu} G_{\sigma}^{\nu}=\partial_{\nu} G_{\sigma}^{\nu}-\Gamma_{\sigma \nu}^{\lambda} G_{\lambda}^{\nu}+\Gamma_{\nu \lambda}^{\nu} G_{\sigma}^{\lambda} \equiv 0
$$

With account of the expression

$$
\Gamma_{\sigma \nu}^{\lambda}=\frac{1}{2} g^{\lambda \rho}\left(\partial_{\sigma} g_{\nu \rho}+\partial_{\nu} g_{\sigma \rho}-\partial_{\rho} g_{\sigma \nu}\right), \quad \partial_{\lambda} \sqrt{g}=\sqrt{g} \Gamma_{\nu \lambda}^{\nu},
$$

one finds

$$
\nabla_{\nu}\left(\sqrt{g} G_{\rho}^{\nu}\right)=\partial_{\nu}\left(\sqrt{g} G_{\rho}^{\nu}\right)+\frac{1}{2} \sqrt{g} G_{\lambda \sigma} \partial_{\rho} g^{\lambda \sigma}=0 .
$$

This identity was obtained by Hilbert in 1915 .

Applying this identity to the invariant $H=R$, where $R$ is the scalar curvature, D. Hilbert obtained the Bianchi idenrity

$$
\nabla_{\nu}\left(R^{\mu \nu}-\frac{1}{2} g^{\mu \nu} R\right) \equiv 0
$$


The detailed account of that is in the main text of this article.

Now we apply Theorem II to the invariant $L$, which depends on $A_{\nu}, \partial_{\lambda} A_{\nu}$, $g_{\mu \nu}, \partial_{\lambda} g_{\mu \nu}$. One has on the basis of $(A .22)$

$$
P_{g}(\sqrt{g} L)=\frac{\delta \sqrt{g} L}{\delta g_{\mu \nu}} \delta_{L} g_{\mu \nu}+\partial_{\lambda} S_{1}^{\lambda}
$$

where

$$
S_{1}^{\lambda}=\frac{\partial \sqrt{g} L}{\partial\left(\partial_{\lambda} g_{\mu \nu}\right)} \delta_{L} g_{\mu \nu}
$$

Likewise

$$
P_{q}(\sqrt{g} L)=\frac{\delta \sqrt{g} L}{\delta A_{\lambda}} \delta_{L} A_{\lambda}+\partial_{\lambda} S_{2}^{\lambda}
$$

where

$$
S_{2}^{\lambda}=\frac{\partial \sqrt{g} L}{\partial\left(\partial_{\lambda} A_{\sigma}\right)} \delta_{L} A_{\sigma} .
$$

One finds from $(A .15),(A .37)$ and $(A .39)$

$$
\int_{\Omega}\left[\frac{\delta \sqrt{g} L}{\delta g_{\mu \nu}} \delta_{L} g_{\mu \nu}+\frac{\delta \sqrt{g} L}{\delta A_{\lambda}} \delta_{L} A_{\lambda}\right] d^{4} x=0 .
$$

Now let us find the Lie variation of the field variable $A_{\lambda}$. According to the transformation law for the vector $A_{\lambda}$ we get

$$
A_{\lambda}^{\prime}\left(x^{\prime}\right)=\frac{\partial x^{\nu}}{\partial x^{\prime \lambda}} A_{\nu}(x)
$$

Thereof we find for transformation $(A .3)$

$$
A_{\lambda}^{\prime}(x+\delta x)=A_{\lambda}(x)-A_{\nu}(x) \partial_{\lambda} \delta x^{\nu} .
$$

Expanding the 1.h.s. into the Taylor series we obtain

$$
\delta_{L} A_{\lambda}=A_{\lambda}^{\prime}(x)-A_{\lambda}(x)=-\delta x^{\nu} \partial_{\nu} A_{\lambda}-A_{\nu}(x) \partial_{\lambda} \delta x^{\nu},
$$

or, in the covariant form,

$$
\delta_{L} A_{\lambda}=-\delta x^{\sigma} \nabla_{\sigma} A_{\lambda}-A_{\sigma} \nabla_{\lambda} \delta x^{\sigma} .
$$


Substituting $(A .27)$ and $(A .45)$ into $(A .41)$ we find

$$
\int_{\Omega} d^{4} x\left[2 \nabla_{\nu}\left(\frac{\delta \sqrt{g} L}{\delta g_{\mu \nu}} g_{\mu \sigma}\right)-\frac{\delta \sqrt{g} L}{\delta A_{\lambda}} \nabla_{\sigma} A_{\lambda}+\nabla_{\lambda}\left(\frac{\delta \sqrt{g} L}{\delta A_{\lambda}} A_{\sigma}\right)\right] \delta x^{\sigma}=0 .
$$

Due to arbitrariness of the transformation vector $\delta x^{\sigma}$ we obtain an identity:

$2 \nabla_{\nu}\left(\frac{\delta \sqrt{g} L}{\delta g_{\mu \nu}} g_{\mu \sigma}\right)=\left(\nabla_{\sigma} A_{\lambda}-\nabla_{\lambda} A_{\sigma}\right) \frac{\delta \sqrt{g} L}{\delta A_{\lambda}}-A_{\sigma} \nabla_{\lambda}\left(\frac{\delta \sqrt{g} L}{\delta A_{\lambda}}\right)$.

According to Hilbert the energy-momentum tensor density is defined by the expression

$$
T^{\mu \nu}=-2 \frac{\delta \sqrt{g} L}{\delta g_{\mu \nu}}
$$

Identity (A.47) assumes the form:

$$
\nabla_{\nu} T_{\sigma}^{\nu}=A_{\sigma} \nabla_{\lambda}\left(\frac{\delta \sqrt{g} L}{\delta A_{\lambda}}\right)+\left(\nabla_{\lambda} A_{\sigma}-\nabla_{\sigma} A_{\lambda}\right) \frac{\delta \sqrt{g} L}{\delta A_{\lambda}}
$$

or

$$
\nabla_{\nu} T_{\sigma}^{\nu}=A_{\sigma} \partial_{\lambda}\left(\frac{\delta \sqrt{g} L}{\delta A_{\lambda}}\right)+\left(\partial_{\lambda} A_{\sigma}-\partial_{\sigma} A_{\lambda}\right) \frac{\delta \sqrt{g} L}{\delta A_{\lambda}}
$$

When the gravitation equations hold, Theorem III leads to the equality

$$
\nabla_{\nu} T_{\sigma}^{\nu}=0
$$

and, hence, identity $(A .50)$ transforms into the equation, which Hilbert designated as Eq.(27) in [6]:

$$
A_{\sigma} \partial_{\lambda}\left(\frac{\delta \sqrt{g} L}{\delta A_{\lambda}}\right)+\left(\partial_{\lambda} A_{\sigma}-\partial_{\sigma} A_{\lambda}\right) \frac{\delta \sqrt{g} L}{\delta A_{\lambda}}=0 .
$$

But this equation holds always due to Hilbert's Axiom I, because

$$
\frac{\delta \sqrt{g} L}{\delta A_{\lambda}}=0
$$




\section{References}

[1] J.Earman and C. Glymour. Einstein and Hilbert: Two Months in the History of General Relativity. Archive for History of Exact Sciences. 19, 291 (1978).

[2] L. Corry, J. Renn and J. Stachel. Belated Decision in the Hilbert-Einstein Priority Dispute. Science. 278, 1270 (1997).

[3] J. Renn and J. Stachel. Hilbert's Foundation of Physics: From a Theory of Everything to a Constituent of General Relativity. Preprint of MaxPlanck-Institut für Wissenschaftsgeschichte. N 118 (1999).

[4] V.P. Vizgin. On the discovery of the gravitational field equations by Einstein and Hilbert: new materials. Physics-Uspekhi. 44, N 12, 1283 (2001).

[5] A. Einstein. Die Feldgleichungen der Gravitation. Sitzunsber. Preuss. Akad. Wiss. 48, 844 (1915). English translation: A. Einstein. The Collected Papers of Albert Einstein (CPAE). 6. (Eds. A.J. Cox et al.)Princeton. N.Y.: Princeton Univ. Press, 1998.

[6] D. Hilbert. Die Grundlagen der Physik. Nachr. Ges. Wiss. Göttingen. 3, 395 (1915).

[7] C.J. Bjerknes. Anticipations of Einstein in the General Theory of Relativity. (XTX INC., Downers Growe, Illinois,) 2003;

Hilbert's Proofs and relative roles of Einstein, Grossmann and Hilbert's contributions are also discussed in the unpublished work of F. Winterberg On "Belated Decision in the Hilbert-Einstein Priority Dispute" by L. Corry, J. Renn and J. Stachel. University of Nevada, Reno, Nevada, 2002.

[8] A. Einstein, M. Grossmann. Entwurf einer Verallgemeinerten Relativätstheorie und Theorie der Gravitation. Z. Math. und Phys. 62, 225 (1913). English translation: CPAE, 4.

[9] A. Einstein. The Collected Papers of Albert Einstein. (Eds. R.Schulmann et al.) Princeton, N.Y.: Princeton Univ. Press. 8, 1998. 
[10] A. Einstein. The meaning of relativity. Princeton Univ. Press. Princeton, N.Y., 1921.

[11] A. Pais. Subtle is the Lord. The Science and Life of Albert Einstein. Oxford University Press, 1982.

[12] D. Hilbert. Die Grundlagen der Physik. Math. Annalen. 92, 1 (1924).

[13] A.Einstein, L. Infeld and B. Hoffmann. Gravitational Equations and Problem of Motion. Ann. Math. 39, 65 (1938).

[14] V.A. Fock. On Motion of Finite Masses in General Theory of Relativity (in Russian). ZhETF. 9, 375 (1939); Rev. Mod. Phys. 29, 325 (1957).

[15] W. Pauli. Relativitätstheorie. Enc. der Math. Wiss. 5, Part 2. B.G. Teubner, Leipzig, 1921.

[16] J.L. Synge. Relativity: The General Theory. North-Holland Publishing Company. Amsterdam, 1960.

[17] S.I. Blinnikov, L.B. Okun', M.I. Vysotskii. Critical Velocities $c / \sqrt{3}$ and $c / \sqrt{2}$ in the general theory of relativity. Physics-Uspekhi.46, N 10, 1099 (2003).

[18] D. Hilbert. Die Grundlagen der Physik (Zweite Mitteilung). Nachr.Ges.Wiss. Göttingen. 1, 53 (1917) .

[19] A.Einstein. Zur allgemeinen Relativitätstheorie. Sitzungsber preuss. Akad. Wiss. 44, 2, 778 (1915). English translation: CPAE, 6.

[20] A. Einstein. Zur allgemeinen Relativitätstheorie. (Nachtrag). Sitzungsber preuss. Akad. Wiss. 46, 2, 799 (1915). English translation: CPAE, 6.

[21] T. Sauer. The Relativity of Discovery: Hilbert's First Note on the Foundations of Physics. Archive for History of Exact Sciences. 53, 529-575 (1999).

[22] A. Einstein. Das hamiltonisches Prinzip und allgemeine Relativitätstheorie. Sitzungsber preuss. Akad. Wiss. 2, 1111 (1916). 